\title{
Systemic Immune-Inflammation Index (SII) is Useful to Predict Survival Outcomes in Patients After Liver Transplantation for Hepatocellular Carcinoma within Hangzhou Criteria
}

\author{
Hongyuan Fu ${ }^{a, b}$ Jun Zheng ${ }^{a, b}$ Jianye Caia Kaining Zenga,b Jia Yao ${ }^{a, b}$ \\ Liang Chen ${ }^{\mathrm{a}, \mathrm{b}}$ Hui Lia,b Jiebin Zhang ${ }^{\mathrm{a}, \mathrm{b}}$ Yingcai Zhang ${ }^{\mathrm{a}, \mathrm{b}}$ Hui Zhao $\mathrm{O}^{\mathrm{a}, \mathrm{b}}$ \\ Yang Yang a,b
}

\begin{abstract}
aDepartment of Hepatic Surgery and Liver Transplantation Center of the Third Affiliated Hospital, Organ Transplantation Institute, Sun Yat-sen University, Organ Transplantation Research Center of Guangdong Province. Guangzhou, bGuangdong Key Laboratory of Liver Disease Research, Key Laboratory of Liver disease biotherapy and Translational Medicine of Guangdong Higher Education Institutes, the Third Affiliated Hospital of Sun Yat-sen University, Guangzhou, China
\end{abstract}

\section{Key Words}

Hepatocellular carcinoma $\cdot$ Systemic immune-inflammatory index $・$ Liver transplantation

\begin{abstract}
Background: There is growing evidence that the systemic immune-inflammation index (SII), a novel prognostic biomarker based on peripheral lymphocyte, neutrophil, and platelet counts, is associated with poor prognosis for several tumors. However, the prognostic value of SII in patients with hepatocellular carcinoma (HCC) who undergo liver transplantation (LT) remains unclear. The aim of this study was to determine the correlation between SII and prognosis in these patients. Methods: This retrospective study involved 150 patients with HCC who underwent LT within the Hangzhou criteria. The optimal cut-off value was determined by receiver-operating characteristic (ROC) curve analysis to stratify the patients into those with a high SII and those with low SII. The Kaplan-Meier method and the Cox proportional hazards model were used to evaluate the prognostic value of SII. Finally, we calculated the area under the ROC curve to compare the prognostic power of SII, platelet-to-lymphocyte ratio (PLR), neutrophil-to-lymphocyte ratio (NLR), and monocyte-to-lymphocyte ratio (MLR). Results: Patients were divided into high SII $(\geq 226)$ and low SII $(<226)$ groups. Five-year overall survival (OS) was lower in the high SII group than in the low SII group (56.1\% vs. $82.4 \%, p=0.002$ ). SII $\geq 226 \times 109 / \mathrm{L}$, maximum tumor size $>5 \mathrm{~cm}$, microvascular invasion, and poor differentiation were independent prognostic factors for OS. However, SII did not predict 5-year recurrencefree survival (high vs. low SII: $64.1 \%$ vs. $78.4 \%, p=0.073$ ). The area under the ROC curve was

$\mathrm{H}$. Fu, J. Zheng and J. Cai contributed equally to this work.

Prof. Yingcai Zhang

Prof. Hui Zhao

Prof. Yang Yang

Dept. of Hepatic Surgery and Liver Transpl. Center of the Third Affiliated Hospital, Organ Transpl. Inst. Sun Yat-sen Univ., Guangzhou (China)

E-Mail zyc88_1120615@sohu.com, zhaohui8@mail.sysu.edu.cn, yysysu@163.com
\end{abstract}




\section{Cellular Physiology Cell Physiol Biochem 2018;47:293-301 \begin{tabular}{ll|l} 
DOI: 10.1159/000489807 & O 2018 The Author(s). Published by S. Karger AG, Basel \\
www.karger.com/cpb
\end{tabular} \\ Fu et al.: The Predictive Value of SII for HCC After LT}

greater for SII than for PLR, NLR, and MLR. Conclusions: Preoperative SII may be a powerful prognostic biomarker in patients with HCC who undergo LT within the Hangzhou criteria. SII is superior to PLR, NLR, and MLR for prediction of OS in these patients.

(C) 2018 The Author(s)

Published by S. Karger AG, Basel

\section{Introduction}

Hepatocellular carcinoma (HCC) is the sixth most common malignant neoplasm and second most common cause of tumor-related deaths worldwide $[1,2]$. HCC is a heterogeneous disease with various etiologies, but chronic infection with a hepatitis virus is the major causative factor. Most cases in developing countries are associated with hepatitis B virus [3] whereas the leading cause in western countries is hepatitis C virus [4]. There are many treatments available for HCC, but liver transplantation (LT) is considered to be the radical treatment of choice, especially in patients with underlying cirrhotic liver disease [5], because it not only removes liver tumors completely but also removes the underlying cirrhotic liver. LT has proven to be the best treatment for selected patients with HCC, but how to select those patients remains controversial, especially in the face of a global organ shortage. The strict Milan criteria devised by Mazzaferro et al. [5]in 1965 have been expanded in recent decades so that more patients with HCC can benefit from LT [6-8]. The Hangzhou criteria based on a Chinese population were proposed by Zheng et al [9]. in 2008 to address the fact that the main etiology of HCC in China is different from that in western countries. According to the Hangzhou criteria, candidates for LT must have (1) a total tumor size $\leq 8 \mathrm{~cm}$ or (2) a total tumor diameter $>8 \mathrm{~cm}$ with a histopathologic grade of I or II and a preoperative alphafetoprotein (AFP) level $\leq 400 \mathrm{ng} / \mathrm{mL}$. In this respect, the Hangzhou criteria are similar to other criteria used to predict the prognosis of HCC patients after LT in that they enroll patients according to factors such as preoperative tumor size and degree of tumor differentiation. However, patients with HCC who meet the Hangzhou criteria often have variable outcomes. Therefore, simple, easily obtained, and dependable prognostic factors are required.

There is abundant evidence that inflammation is associated with carcinogenesis and tumor progression [10-12]. Furthermore, a growing number of studies have demonstrated that circulating immune-inflammatory cells, such as platelets, neutrophils, and lymphocytes, play a prominent role in promoting proliferation, invasion, and migration of cancer cells by changing the tumor microenvironment. These cells are thought to affect outcome in various ways, including shielding circulating tumor cells from destruction by the immune system [13], secreting cytokines and chemokines $[14,15]$, and inducing cytotoxic cell death $[16,17]$.

Much research effort has been devoted to identifying indicators based on these immuneinflammatory circulating cells that can predict prognosis in immune-mediated diseases. In the last decade, more and more evidence has emerged showing that the neutrophil-to-lymphocyte ratio (NLR), platelet-to-lymphocyte ratio (PLR), and monocyte-to-lymphocyte ratio (MLR) could be useful for predicting prognosis in various types of cancer, including HCC [18-20]. However, these indicators involve only two types of circulating immune-inflammatory cells and might not accurately reflect the inflammatory and immune responses in the host body. A novel indicator known as the systemic immune-inflammation index (SII) was developed recently and has been demonstrated to be an effective and powerful prognostic indicator for several types of tumors, including HCC, colorectal cancer, and small cell lung cancer [21-23]. SII differs from other indicators in that it combines peripheral lymphocyte, neutrophil, and platelet counts. This combination could have a stronger prognostic ability in some diseases. However, the relationship between preoperative SII and prognosis in patients with HCC after LT remains unclear. The aim of this study was to investigate the prognostic value of SII in patients undergoing LT for HCC within the Hangzhou criteria and to compare its prognostic ability with that of the NLR, PLR, and MLR 


\section{Cellular Physiology Cell Physiol Biochem 2018;47:293-301

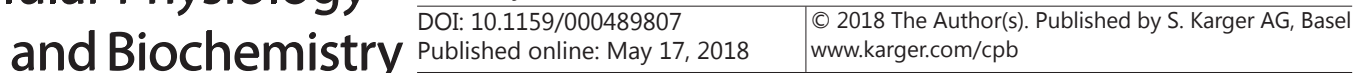

Fu et al.: The Predictive Value of SII for HCC After LT

\section{Materials and Methods}

\section{Patients}

This retrospective study involved patients who underwent LT at the Third Affiliated Hospital of Sun Yat-sen University (Guangdong, China) since October 2003. Handwritten and electronic medical records were screened carefully until December 2016. Patients were eligible for inclusion in the study if they met the following criteria: recipient age older than 18years; a histopathologic diagnosis of HCC that met the Hangzhou criteria [9]; no pre-transplant radiofrequency ablation, liver resection, or transarterial chemoembolization; no pre-transplant sepsis or massive gastrointestinal hemorrhage; no history of taking hematopoietic agents within the month before LT; and availability of complete and authentic clinicopathologic and follow-up data. One hundred and fifty patients who underwent LT between October 2003 and December 2015 met these criteria and were enrolled.

\section{Follow-up}

After LT, the patients underwent regular follow-up in our outpatient clinics. A physical examination was routinely performed at all follow-up appointments. Serum AFP levels were measured and hepatobiliary ultrasonography was performed at monthly intervals in the first year and then every 3 months thereafter if no recurrence was suspected. Plain/enhanced abdominal computed tomography (CT) scans or magnetic resonance imaging was performed every 3 months in the first 2 years, and then every 6 months thereafter. An enhanced CT scan of the lung, positron emission tomography-CT scan, or a bone scan was carried out immediately when necessary if a metastasis was suspected. Overall survival (OS) was calculated from the time of LT to the date of death or last follow-up. Recurrence-free survival (RFS) was defined as the interval between LT and recurrence or last tumor evaluation/last follow-up or death. The last follow-up was performed at the end of March 2017.

\section{Study design and data collection}

We obtained neutrophil, lymphocyte, monocyte, and platelet counts from each patient's peripheral venous blood sample during the week before LT, usually in the morning before surgery. Cell counts in these morning fasting venous blood samples were measured using a fully automatic hematology analyzer. Information on other potential prognostic factors was also collected and evaluated, including demographic data (including age and sex), clinical and laboratory data obtained within the week before surgery (including AFP, albumin, and HBV DNA), preoperative imaging data (tumor size, number of tumors, and macrovascular invasion), and postoperative histopathologic data (microvascular invasion and tumor cell differentiation grade). The relevant indicators were calculated as follows: SII = P (absolute platelet count) $\times \mathrm{N}$ (absolute neutrophil count)/L (absolute lymphocyte count), PLR = P (absolute platelet count)/L (lymphocyte count), $\mathrm{NLR}=\mathrm{N}$ (absolute neutrophil count)/L (absolute lymphocyte count), and MLR = M (absolute monocyte count)/L (absolute lymphocyte count).

\section{Statistical analysis}

Patients' baseline characteristics are shown as the mean \pm standard deviation for normally distributed continuous variables and as the median and interquartile range for non-normally distributed continuous variables. Categorical variables are reported as proportions. Receiver-operating characteristic (ROC) curves were plotted to determine the optimal cut-off values for SII, PLR, NLR, and MLR. The independent samples t-test, Pearson's chi-squared test, and Fisher's exact test were used to assess the correlations between SII and other clinicopathologic characteristics as appropriate. Univariate and multivariate Cox proportional hazards regression were used to identify independent risk factors. The survival curves were plotted using the Kaplan-Meier method and tested by the log-rank statistic. Finally, we plotted the ROC curves and calculated the area under the ROC curve to compare the discriminative ability of SII, PLR, NLR, and MLR as inflammatory indicators. A p-value $<0.05$ was accepted to be statistically significant. All p-values were based on two-sided testing. The statistical analyses were performed using SPSS version 22.0 (IBM Corp., Armonk, NY), GraphPad Prism version 5.0 (GraphPad Software, La Jolla, CA), and MedCalc version 13.0 (Mariakerke, Belgium). 


\begin{tabular}{|c|c|c|}
\hline \multirow{2}{*}{$\begin{array}{l}\text { Cellular Physiology } \\
\text { and Biochemistry }\end{array}$} & \multicolumn{2}{|c|}{ Cell Physiol Biochem 2018;47:293-301 } \\
\hline & $\begin{array}{l}\text { DOI: 10.1159/000489807 } \\
\text { Published online: May 17, } 2018\end{array}$ & $\begin{array}{l}\text { O } 2018 \text { The Author(s). Published by S. Karger AG, Basel } \\
\text { www.karger.com/cpb }\end{array}$ \\
\hline
\end{tabular}

Ethics

The study was approved by the Institutional Review Board of the Third Affiliated Hospital of Sun Yat-sen University and adhered to the tenets of the Declaration of Helsinki. Written informed consent was obtained from all patients.

\section{Results}

Patient demographics and outcomes

The 150 patients in the study were 8 (5.3\%) women and 142 (94.7\%) men (Table 1). Median age was 51.0 (interquartile range 45.0-59.0) years. During a median follow-up of 41 months, 39 patients died and 37 were confirmed to have a tumor recurrence. The 1-, 3 - and 5-year OS rates were $89.9 \%, 76.6 \%$, and $69.9 \%$, respectively; the 1-, 3- and 5-year RFS rates were $86.1 \%$, $75.0 \%$, and $71.6 \%$, respectively.

Comparison of variables when patients were stratified by SII

We plotted a ROC curve to determine the optimal cut-off SII for predicting survival after LT for HCC (Fig. 1). The area under the ROC curve was 0.632 and the Youden index was highest when SII was $226 \times 10^{9} / \mathrm{L}$; the sensitivity and specificity were $69.2 \%$ and $58.6 \%$, respectively. Therefore, we divided the patients into a high SII group ( $\left.\geq 226 \times 10^{9} / \mathrm{L}, \mathrm{n}=73\right)$ and a low SII group $(<$ $\left.226 \times 10^{9} / \mathrm{L}, \mathrm{n}=77\right)$. Demographic and clinicopathologic characteristics were compared between the two groups. Patients with high SII were more likely to have larger tumor size ( $p=0.043)$, greater total tumor volume $(p=0.034)$, higher AFP level $(p=0.047)$, and poor differentiation $(p=0.040)$. No significant differences in the other variables were found between the groups (Table 2).

Table 2. The relationship between SII and clinical characteristics in HCC patients. HCC: hepatocellular carcinoma; SII:immune-inflammation index; TTV: total tumor volume; AFP: $\alpha$-fetoprotein; MVI: mirco-vascular invasion
Table 1. Baseline Characteristics in HCC patients

\begin{tabular}{lc} 
Characteristic & Values \\
\hline Gender & $142(94.7 \%)$ \\
Male & $8(5.3 \%)$ \\
Female & $51.0(45.0-59.0)$ \\
Age(years) & \\
Child-Pugh Class & $81(54.0 \%)$ \\
A & $48(32.0 \%)$ \\
B & $21(14.0 \%)$ \\
C & \\
HBsAg & $139(92.7 \%)$ \\
Positive & $11(7.3 \%)$ \\
Negative & $1(1-2)$ \\
Tumor No. (n) & $3.1(2.3-4.8)$ \\
Largest Tumor Size(cm) & $4.2(3.0-6.1)$ \\
Total Tumor Size(cm) & $18.8(7.9-65.4)$ \\
TTV(cm 3$)$ & $37.1(5.9-269.0)$ \\
AFP(ng/ml) & \\
MVI & $24(16.0 \%)$ \\
Positive & $126(84.0 \%)$ \\
Negative & \\
Differentiation & $31(20.7 \%)$ \\
Well & $119(79.3 \%)$ \\
Moderate/Poor & $2.49(1.67-3.61)$ \\
Neutrophil(10 $/ \mathrm{L})$ & $1.03(0.73-1.44)$ \\
Lymphocyte(10 $/ \mathrm{L})$ & $0.34(0.23-0.50)$ \\
Monocyte(10 $/ \mathrm{L})$ & $92.0(54.8-151.3)$ \\
Platelet(10 $/$ L) & $210.1(109.7-427.0)$ \\
SII & $86.2(58.8-124.0)$ \\
PLR & $2.26(1.40-3.79)$ \\
NLR & $0.34(0.21-0.53)$ \\
MLR & \\
\hline &
\end{tabular}

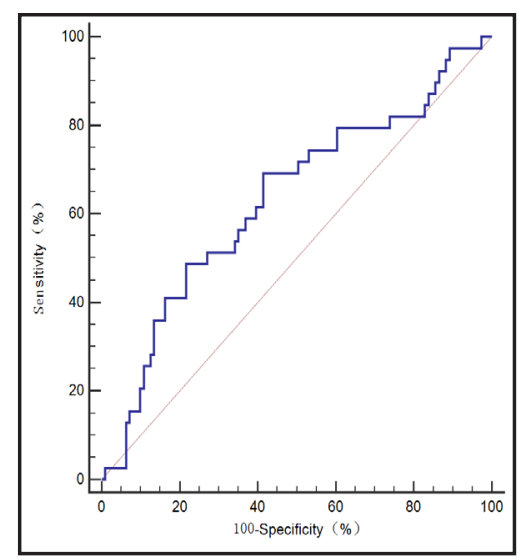

Fig. 1. ROC curve for the SII values to predict HCC survival after LT within Hangzhou Criteria.

\begin{tabular}{|c|c|c|c|c|}
\hline Variables & SII $<226(n=77)$ & SII $\geq 226(n=73)$ & tor $\chi^{2}$ or $z$ & $P$ value \\
\hline Gender(Male) & $75(97.4 \%)$ & $67(91.8 \%)$ & 1.364 & 0.243 \\
\hline Age, yr (mean) & 51.38 & 51.01 & 0.222 & 0.824 \\
\hline Child-Pugh Class & & & -1.953 & 0.051 \\
\hline$\Lambda$ & $35(15.5 \%)$ & $16(63.0 \%)$ & & \\
\hline B & $30(39.0 \%)$ & $18(24.7 \%)$ & & \\
\hline $\mathrm{C}$ & $12(15.6 \%)$ & $9(12.3 \%)$ & & \\
\hline $\operatorname{HBsAg}(+)$ & $74(96.1 \%)$ & $65(89.0 \%)$ & 2.751 & 0.097 \\
\hline Tumor No. $(n>3)$ & $6(7.8 \%]$ & $2(2.7 \%)$ & 1.026 & 0.311 \\
\hline Largest Tumor Size $(>5 \mathrm{~cm})$ & $10(13.0 \%)$ & $19(26.0 \%)$ & 4.086 & $0.043^{*}$ \\
\hline Total Tumor Size $(>7 \mathrm{~cm})$ & $11(14.3 \%)$ & $16(21.9 \%)$ & 1.479 & 0.224 \\
\hline $\operatorname{TTV}\left(>20 \mathrm{~cm}^{3}\right)$ & $32(41.6 \%)$ & $43(58.9 \%)$ & 4.510 & $0.034^{*}$ \\
\hline $\operatorname{AFP}(\geq 400)$ & $11(14.3 \%)$ & $20(27.4 \%)$ & 3.929 & $0.047^{*}$ \\
\hline MVI(+) & $14(18.2 \%)$ & $10(13.7 \%)$ & 0.560 & 0.454 \\
\hline Differentiation(M/P) & $56(72.7 \%)$ & $63(86.3 \%)$ & 4.211 & $0.040^{*}$ \\
\hline
\end{tabular}




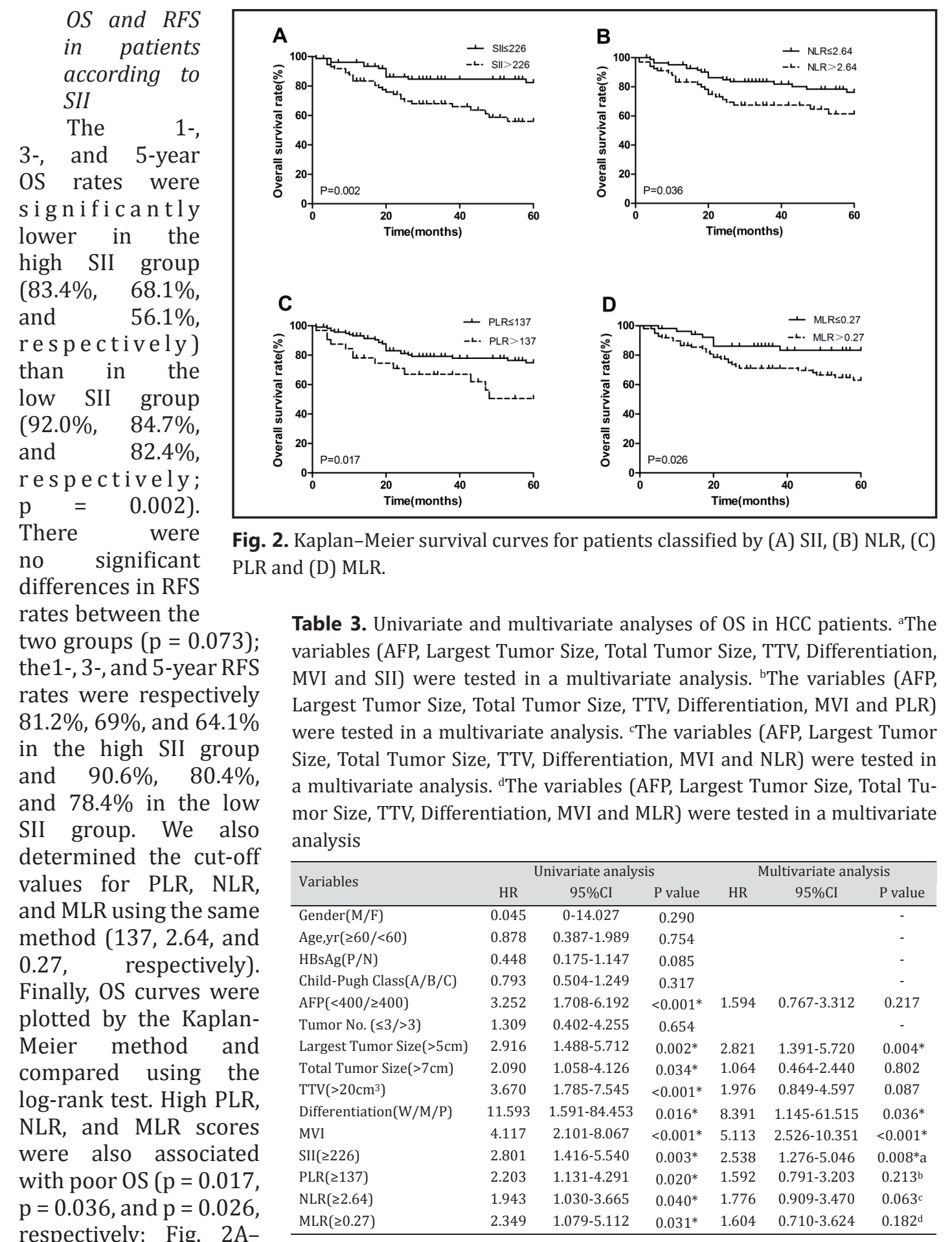
2D).

\section{Univariate and multivariate survival analyses}

Univariate analysis revealed largest tumor size, total tumor size, microvascular invasion, AFP level, tumor cell differentiation, total tumor volume, SII, PLR, NLR, and MLR to be significant prognostic factors for OS. To avoid multicollinearity, we conducted multivariate analysis using four models separately. Each multivariate model included only one immuneinflammatory indicator (SII, PLR, NLR, or MLR) or other significant predictor identified in 


\section{Cellular Physiology Cell Physiol Biochem 2018;47:293-301 \begin{tabular}{l|l} 
DOI: 10.1159/000489807 & and Biochemistry \\
Published online:May 17, 2018 & $\begin{array}{l}\text { 2 } 218 \text { The Author(s). Published by S. Karger AG, Basel } \\
\text { www.karger.com/cpb }\end{array}$
\end{tabular} \\ Fu et al.: The Predictive Value of SII for HCC After LT}

Fig. 3. Predictive effect of the SII (categorical) was compared with PLR (categorical), NLR (categorical) and MLR (categorical) by ROC curves in 3-years (A) and 5-years (B) survival. Predictive effect of the SII (continuous) was compared with PLR (continuous), NLR (continuous) and MLR (continuous) by ROC curves in 3-years (C) and 5-years (D) survival.

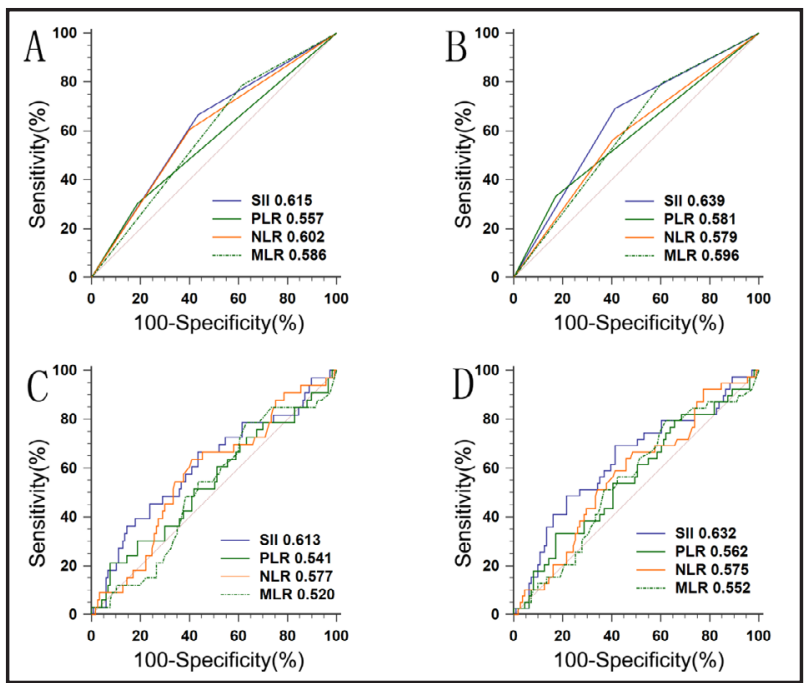

univariate analysis. After multivariate analysis, SII $\geq 226 \times 10^{9} / \mathrm{L}$ (hazard ratio [HR] 2.538, 95\% confidence interval [CI] 1.276-5.046; $\mathrm{p}=0.008$ ), microvascular invasion (HR 5.113, 95\% CI 2.526-10.351; $\mathrm{p}<0.001$ ), poor tumor cell differentiation (HR 8.391, 95\% CI 1.14561.515; $\mathrm{p}=0.036$ ), and maximum tumor size $>5 \mathrm{~cm}$ (HR 2.821, 95\% CI 1.391-5.720; $\mathrm{p}=$ 0.002 ) were revealed to be independent predictors of OS after LT in patients with HCC, but PLR, NLR, and MLR were not ( $p=0.213, p=0.063$, and $p=0.182$, respectively; Table 3 ). We also performed univariate and multivariate survival analyses for RFS and did not identify any immune-inflammatory indicator as an independent predictor of RFS.

\section{Comparison of prognostic significance of SII, NLR, PLR, and MLR}

ROC curve analysis was performed after 3 and 5 years of follow-up. The discriminative ability of SII, PLR, NLR, and MLR were compared using the area under the ROC curve. At the 3-year follow-up, of all the factors tested, SII had the largest area under the ROC curve (Fig. 3). Furthermore, at the 5-year follow-up, the area under the ROC curve for SII was still higher than those for PLR, NLR, and MLR for predicting survival (Fig. 3A, 3B). Similar results were found when SII was treated as a continuous variable (Fig. 3C, 3D), indicating that SII was better than NLR, PLR and MLR for predicting OS in HCC patients after LT.

\section{Discussion}

To our knowledge, no previous study has investigated the predictive value of SII after LT in patients with HCC. This study is the first to demonstrate an association between SII and clinical outcomes in these patients and provides a new option for predicting their prognosis and for selecting appropriate recipients for LT. As mentioned earlier, SII is widely accepted to be useful for predicting the prognosis of several types of cancer [21-23], but the optimal cut-off values for SII remain controversial. However, it is known that these values may be treatment-dependent even in patients with the same disease. For example, SII > $330 \times 10^{9} / \mathrm{L}$ was reported to be associated with poorer prognosis after curative resection of HCC [21], whereas Casadei et al. [24]found that SII of $360 \times 10^{9} / \mathrm{L}$ was the optimal cut-off value in patients with advanced HCC receiving sorafenib. ROC curve analysis in the present study identified the optimal cut-off value for SII as $230 \times 10^{9} / \mathrm{L}$ after LT in patients with HCC. The patients were then grouped according to whether they had a high SII or a low SII. Our results showed that a higher preoperative SII $\left(\geq 226 \times 10^{9} / \mathrm{L}\right)$ was associated with larger tumor size, a higher AFP level, and poor pathologic differentiation. We demonstrated that SII was an independent prognostic factor for OS and RFS in patients who underwent LT for HCC. 
We also concluded that SII was a more powerful prognostic indicator than other systemic inflammatory indicators, such as PLR and NLR. Given that it is inexpensive, is easily obtained, and has good predictive accuracy, SII could be a promising biomarker for predicting the prognosis in patients after LT for HCC within the Hangzhou criteria.

Although SII was demonstrated to be correlated with prognosis of HCC patients after LT, the mechanism has not been elucidated. The reason might be that SII predicts prognosis from levels of inflammatory and immune response markers in the host. A higher SII indicates relative hyperthrombocytemia, neutrophilia, and lymphopenia, and an imbalance of neutrophils, platelets, and lymphocytes leads to disorders in the host's immune system. Vascular endothelial growth factor (VEGF) plays an important role in angiogenesis and tumor progression [25] and can be secreted by both neutrophils and platelets. Elevated levels of VEGF promote tumor progression. Furthermore, lymphocytes are important immune cells that are considered to promote tumor control by secreting cytokines and inducing cytotoxic cell death [26]. A decreased lymphocyte level will impair the host's immunity and encourage tumor progression. Several studies have demonstrated that tumors are usually infiltrated by various lymphocytes, and tumor-infiltrating lymphocyte levels correlate with the progression and aggressiveness of a tumor [27-29]. There were strong links between circulating lymphocytes and tumor-infiltrating lymphocytes. Targeted agents that regulate inflammation and immunity may improve the prognosis of patients with immune-mediated diseases. Aspirin was demonstrated to be helpful in protecting patients with chronic hepatitis from HCC [30] and could also be effective as secondary prevention in patients with colorectal cancer [31]. Thymosin has shown antitumor effects via enhancement of the Th1 immune response [32]. In the future, HCC patients might also benefit from drugs that target inflammation and immunity.

This study has several limitations. First, SII may be affected by many factors, including unidentified acute infection and gastrointestinal hemorrhage. Second, the majority of patients enrolled in this study had a background of hepatitis B infection, while hepatitis C virus-associated HCC is more common in western countries. It should be noted that SII is a dynamic index that changes during the course of a disease, and changes in SII during the treatment course may provide more information about the host's inflammatory and immune response status. Therefore, further study is needed to observe the dynamic changes in SII before and after LT. Furthermore, SII may have a role in mediating cytokines, so detecting cytokine levels in the patient's blood or tissues may help to reveal the possible mechanism. Subsequent studies should focus on the relationship between the patient's cytokine levels and the prognosis. Finally, this study had a retrospective design and included a relatively small number of patients. Further prospective studies in larger samples are needed to verify the prognostic value of SII in HCC patients after LT.

In conclusion, the results of this study indicate that patients with HCC meeting the Hangzhou criteria and an elevated preoperative SII have poorer OS after LT. Because of its attributes of comprehensiveness, simplicity, easy determination, and low cost, SII appears to be a promising tool for assessing prognosis after LT in patients with HCC who meet the Hangzhou criteria.

\section{Abbreviations}

OS (overall survival); HCC (hepatocellular carcinoma); TTV (total tumor volume); AFP ( $\alpha$-fetoprotein); MVI (mirco-vascular invasion); SII (immune-inflammation index); PLR (platelet lymphocyte ratio); NLR (neutrophil lymphocyte ratio); MLR (monocyte lymphocyte ratio).

\section{Acknowledgements}

This work was supported by the grants from the National Natural Science Foundation of China $(81370575,81570593,81770648)$, Guangdong Natural Science Foundation 


\section{Cellular Physiology Cell Physiol Biochem 2018;47:293-301 \begin{tabular}{l|l|l} 
and BOI: 10.1159/000489807 & $\begin{array}{l}\text { C) } 2018 \text { The Author(s). Published by S. Karger AG, Basel } \\
\text { www.karger.com/cpb }\end{array}$
\end{tabular}}

Fu et al.: The Predictive Value of SII for HCC After LT

(2015A030312013), Sci-tech Research Development Program of Guangdong province (2017A020215023), Sci-tech Research Development Program of Guangzhou city (158100076), Sun Yat-Sen University Clinical Research 5010 Program (2014006); and Young Teacher Development Program of Sun Yat-Sen University (17ykpy57).

\section{Disclosure Statement}

The authors who have been engaged in this clinical research declared that they did not have any conflict of interests.

\section{References}

1 Ferlay J, Soerjomataram I, Dikshit R, Eser S, Mathers C, Rebelo M, Parkin DM, Forman D, Bray F: Cancer incidence and mortality worldwide: sources, methods and major patterns in GLOBOCAN 2012. Int J Cancer 2015;136:E359-386.

-2 Jemal A, Bray F, Center MM, Ferlay J, Ward E, Forman D: Global cancer statistics. CA Cancer J Clin 2011;61:69-90.

3 Trepo C, Chan HL, Lok A: Hepatitis B virus infection. Lancet 2014;384:2053-2063.

4 Westbrook RH, Dusheiko G: Natural history of hepatitis C. J Hepatol 2014;61:S58-68.

5 Mazzaferro V, Regalia E, Doci R, Andreola S, Pulvirenti A, Bozzetti F, Montalto F, Ammatuna M, Morabito A, Gennari L: Liver transplantation for the treatment of small hepatocellular carcinomas in patients with cirrhosis. N Engl J Med 1996;334:693-699.

6 Yao FY, Ferrell L, Bass NM, Watson JJ, Bacchetti P, Venook A, Ascher NL, Roberts JP: Liver transplantation for hepatocellular carcinoma: expansion of the tumor size limits does not adversely impact survival. Hepatology 2001;33:1394-1403.

7 Freeman RB, Mithoefer A, Ruthazer R, Nguyen K, Schore A, Harper A, Edwards E: Optimizing staging for hepatocellular carcinoma before liver transplantation: A retrospective analysis of the UNOS/OPTN database. Liver Transpl 2006;12:1504-1511.

8 Mazzaferro V, Llovet JM, Miceli R, Bhoori S, Schiavo M, Mariani L, Camerini T, Roayaie S, Schwartz ME, Grazi GL, Adam R, Neuhaus P, Salizzoni M, Bruix J, Forner A, De Carlis L, Cillo U, Burroughs AK, Troisi R, Rossi M, Gerunda GE, Lerut J, Belghiti J, Boin I, Gugenheim J, Rochling F, Van Hoek B, Majno P: Predicting survival after liver transplantation in patients with hepatocellular carcinoma beyond the Milan criteria: a retrospective, exploratory analysis. Lancet Oncol 2009;10:35-43.

-9 Zheng SS, Xu X, Wu J, Chen J, Wang WL, Zhang M, Liang TB, Wu LM: Liver transplantation for hepatocellular carcinoma: Hangzhou experiences. Transplantation 2008;85:1726-1732.

10 Diakos CI, Charles KA, McMillan DC, Clarke SJ: Cancer-related inflammation and treatment effectiveness. Lancet Oncol 2014;15:e493-503.

-11 Coussens LM, Werb Z: Inflammation and cancer. Nature 2002;420:860-867.

-12 Grivennikov SI, Greten FR, Karin M: Immunity, inflammation, and cancer. Cell 2010;140:883-899.

-13 Labelle M, Begum S, Hynes R0: Direct signaling between platelets and cancer cells induces an epithelialmesenchymal-like transition and promotes metastasis. Cancer Cell 2011;20:576-590.

14 Gregory AD, Houghton AM: Tumor-associated neutrophils: new targets for cancer therapy. Cancer Res 2011;71:2411-2416.

15 Prieto J, Melero I, Sangro B: Immunological landscape and immunotherapy of hepatocellular carcinoma. Nat Rev Gastroenterol Hepatol 2015;12:681-700.

16 Mantovani A, Allavena P, Sica A, Balkwill F: Cancer-related inflammation. Nature 2008;454:436-444.

17 Minami T, Minami T, Shimizu N, Yamamoto Y, De Velasco M, Nozawa M, Yoshimura K, Harashima N, Harada M, Uemura H: Identification of Programmed Death Ligand 1-derived Peptides Capable of Inducing Cancerreactive Cytotoxic T Lymphocytes From HLA-A24+ Patients With Renal Cell Carcinoma. J Immunother 2015;38:285-291.

18 Halazun KJ, Hardy MA, Rana AA, Woodland DCt, Luyten EJ, Mahadev S, Witkowski P, Siegel AB, Brown RS, Jr., Emond JC: Negative impact of neutrophil-lymphocyte ratio on outcome after liver transplantation for hepatocellular carcinoma. Ann Surg 2009;250:141-151. 


\section{Cellular Physiology Cell Physiol Biochem 2018;47:293-301 \begin{tabular}{l|l} 
DOI: 10.1159/000489807 & Ond Biochemistry 2018 The Author(s). Published by S. Karger AG, Basel \\
wwww.karger.com/cpb
\end{tabular} \\ Fu et al.: The Predictive Value of SII for HCC After LT}

19 Lai Q, Castro Santa E, Rico Juri JM, Pinheiro RS, Lerut J: Neutrophil and platelet-to-lymphocyte ratio as new predictors of dropout and recurrence after liver transplantation for hepatocellular cancer. Transpl Int 2014;27:32-41.

20 Tang T, Liao R, Li J, Du CY: [Analysis of prognostic factors of patients with hepatocellular carcinoma after radical resection]. Zhonghua Wai Ke Za Zhi 2016;54:439-443.

21 Wang Y, Li M, Long J, Shi XY, Li Q Chen J, Tong WM, Jia JD, Huang J: Clinical significance of increased expression of Nijmegen breakage syndrome gene (NBS1) in human primary liver cancer. Hepatol Int 2014;8:250-259.

-22 Passardi A, Scarpi E, Cavanna L, Dall'Agata M, Tassinari D, Leo S, Bernardini I, Gelsomino F, Tamberi S, Brandes AA, Tenti E, Vespignani R, Frassineti GL, Amadori D, De Giorgi U: Inflammatory indexes as predictors of prognosis and bevacizumab efficacy in patients with metastatic colorectal cancer. Oncotarget 2016;7:33210-33219.

23 Hong X, Cui B, Wang M, Yang Z, Wang L, Xu Q: Systemic Immune-inflammation Index, Based on Platelet Counts and Neutrophil-Lymphocyte Ratio, Is Useful for Predicting Prognosis in Small Cell Lung Cancer. Tohoku J Exp Med 2015;236:297-304.

-24 Casadei Gardini A, Scarpi E, Faloppi L, Scartozzi M, Silvestris N, Santini D, de Stefano G, Marisi G, Negri FV, Foschi FG, Valgiusti M, Ercolani G, Frassineti GL: Immune inflammation indicators and implication for immune modulation strategies in advanced hepatocellular carcinoma patients receiving sorafenib. Oncotarget 2016;7:67142-67149.

25 Bambace NM, Holmes CE: The platelet contribution to cancer progression. J Thromb Haemost 2011;9:237249.

-26 Ferrone C, Dranoff G: Dual roles for immunity in gastrointestinal cancers. J Clin Oncol 2010;28:4045-4051.

27 Chew V, Chen J, Lee D, Loh E, Lee J, Lim KH, Weber A, Slankamenac K, Poon RT, Yang H, Ooi LL, Toh HC, Heikenwalder M, Ng IO, Nardin A, Abastado JP: Chemokine-driven lymphocyte infiltration: an early intratumoural event determining long-term survival in resectable hepatocellular carcinoma. Gut 2012;61:427-438.

28 Jiang D, Liu Y, Wang H, Wang H, Song Q, Sujie A, Huang J, Xu Y, Zeng H, Tan L, Hou Y, Xu C: Tumour infiltrating lymphocytes correlate with improved survival in patients with esophageal squamous cell carcinoma. Sci Rep 2017;7:44823.

29 West NR, Kost SE, Martin SD, Milne K, Deleeuw RJ, Nelson BH, Watson PH: Tumour-infiltrating FOXP3(+) lymphocytes are associated with cytotoxic immune responses and good clinical outcome in oestrogen receptor-negative breast cancer. Br J Cancer 2013;108:155-162.

-30 Sahasrabuddhe VV, Gunja MZ, Graubard BI, Trabert B, Schwartz LM, Park Y, Hollenbeck AR, Freedman ND, McGlynn KA: Nonsteroidal anti-inflammatory drug use, chronic liver disease, and hepatocellular carcinoma. J Natl Cancer Inst 2012;104:1808-1814.

-31 Bains SJ, Mahic M, Myklebust TA, Smastuen MC, Yaqub S, Dorum LM, Bjornbeth BA, Moller B, Brudvik KW, Tasken K: Aspirin As Secondary Prevention in Patients With Colorectal Cancer: An Unselected PopulationBased Study. J Clin Oncol 2016;34:2501-2508.

-32 Sjogren MH: Thymalfasin: an immune system enhancer for the treatment of liver disease. J Gastroenterol Hepatol 2004;19:S69-72. 